\title{
oxLDL promotes podocyte migration by regulating CXCL16, ADAM10 and ACTN4
}

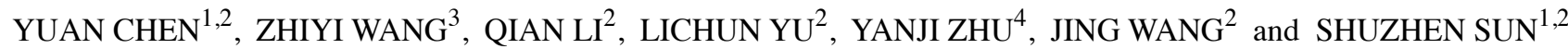 \\ ${ }^{1}$ Department of Pediatrics, Shandong Provincial Hospital, Cheeloo College of Medicine, Shandong University; \\ Departments of ${ }^{2}$ Pediatrics and ${ }^{3}$ Hepatobiliary Surgery, Shandong Provincial Hospital Affiliated \\ to Shandong First Medical University, Jinan, Shandong 250021; ${ }^{4}$ Department of Pediatrics, \\ People's Hospital of Rizhao, Rizhao, Shandong 276800, P.R. China
}

Received September 14, 2019; Accepted May 22, 2020

DOI: $10.3892 / \mathrm{mmr} .2020 .11292$

\begin{abstract}
Nephrotic syndrome (NS) is one of the most common causes of chronic kidney disease in the pediatric population. Hyperlipidemia is one of the main features of NS. The present study investigated the role of CXC motif chemokine ligand 16 (CXCL16) and ADAM metallopeptidase domain 10 (ADAM10) in oxidized low-density lipoprotein (oxLDL)-stimualted podocytes and the underlying mechanisms. CXCL16 and ADAM10 expression levels in oxLDL-treated podocytes were measured via reverse transcription-quantitative PCR and western blotting. Cell migration assays were conducted to assess the migration of oxLDL-treated podocytes. CXCL16 or ADAM10 overexpression and knockdown assays were conducted. The results indicated that oxLDL stimulation increased ADAM10 and CXCL16 expression levels, and enhanced podocyte migration compared with the control group. Moreover, CXCL16 and ADAM10 overexpression significantly increased podocyte migration and the expression of actinin- $\alpha 4$ (ACTN4) compared with the control groups. By contrast, CXCL16 and ADAM10 knockdown significantly reduced podocyte migration and the expression of ACTN4 compared with the control groups. The results suggested that oxLDL promoted podocyte migration by regulating CXCL16 and ADAM10 expression, as well as by modulating the actin cytoskeleton. Therefore, CXCL16 and ADAM10 may serve as novel therapeutic targets for primary nephrotic syndrome in children.
\end{abstract}

Correspondence to: Professor Shuzhen Sun, Department of Pediatrics, Shandong Provincial Hospital, Cheeloo College of Medicine, Shandong University, 324 Jing Wu Road, Jinan, Shandong 250021, P.R. China E-mail: shuzhensun 001@126.com

Abbreviations: oxLDL, oxidized low-density lipoprotein; ADAM10, ADAM metallopeptidase domain 10; CXCL16, CXC motif chemokine ligand 16; MPC5, mouse podocyte clone-5; ACTN4, actinin- $\alpha 4$; CXCR6, CXC motif chemokine receptor 6; shRNA, short hairpin RNA

Key words: oxLDL, podocytes, migration, CXCL16, ADAM10, ACTN4

\section{Introduction}

Nephrotic syndrome (NS) is one of the most common causes of chronic kidney disease in the pediatric population (1). NS is primarily characterized by extensive proteinuria, hypoalbuminemia, hyperlipidemia and edema, resulting from disruption of the glomerular filtration barrier (2). A kidney biopsies taken from children with NS are often associated with podocyte foot process effacement, whereas kidney biopsies taken from healthy children are not (3).

Previous studies have demonstrated that oxidized low-density lipoprotein (oxLDL) may cause podocyte damage $(4,5)$; however, the underlying mechanism is not completely understood. Evidence has suggested that oxLDL promotes the migration of monocytes, smooth muscle cells and bone marrow-derived mesenchymal stem cells (6-8); however, whether oxLDL results in podocyte migration, as well as its associated mechanism, requires further investigation.

CXC motif chemokine ligand 16 (CXCL16) is a scavenger receptor that exists in two distinct forms: A membrane-bound form and a soluble form (9). Membrane-bound CXCL16 can be released as the soluble form upon digestion by ADAM metallopeptidase domain (ADAM) proteins, specifically ADAM10 and ADAM17 (10). The membrane-bound form of CXCL16 binds to and internalizes oxLDL to promote the adhesion of cells expressing CXC motif chemokine receptor 6 (CXCR6), the only known receptor of CXCL16 (11). By contrast, soluble CXCL16 functions as a chemoattractant to promote the migration of CXCR6-expressing cells (12).

Previous studies have demonstrated that CXCL16 and ADAM10 are expressed in the kidney $(11,13,14)$, and Gutwein et al (15) reported that CXCL16 and ADAM10 are constitutively expressed in podocytes. However, a limited number of studies have investigated the role of CXCL16 and ADAM10 in proteinuria and the progression of glomerular diseases.

Schramme et al (16) revealed that inhibition of ADAM10 and CXCL16 expression in mesangial cells results in a significant reduction in cell proliferation and migration. Increasing evidence has also demonstrated that soluble CXCL16 promotes cancer cell migration in vitro (12). Collectively, the results of the aforementioned studies suggest that CXCL16 
and ADAM10 may promote podocyte migration and may be involved in the development of primary NS by mediating lipid stimulation and inflammatory responses (17).

The present study investigated the expression of ADAM10 and CXCL16 in an oxLDL-stimulated conditionally immortalized mouse podocyte cell line (MPC5). Additionally, the effect of oxLDL on cell migration was analyzed by performing functional assays. The results suggested that oxLDL stimulation increased the expression of ADAM10 and CXCL16, and promoted podocyte migration. Furthermore, the effects of CXCL16 and ADAM10 overexpression and knockdown in MPC5 cells were investigated, and the expression of actinin- $\alpha 4$ (ACTN4) was detected via western blotting.

\section{Materials and methods}

Cell culture. The conditionally immortalized mouse podocyte cell line MPC5 was a gift from Professor Rong Wang (Department of Nephrology, Shandong Provincial Hospital Affiliated to Shandong University). Cells were cultured in RPMI-1640 medium (Gibco; Thermo Fisher Scientific, Inc.) containing 10\% FBS (Gibco; Thermo Fisher Scientific, Inc.), $100 \mathrm{U} / \mathrm{ml}$ penicillin, $100 \mu \mathrm{g} / \mathrm{ml}$ streptomycin and $10 \mathrm{U} / \mathrm{ml}$ mouse interferon- $\gamma$ (IFN- $\gamma$; cat. no. C600059; Sangon Biotech Co., Ltd.) at $33^{\circ} \mathrm{C}$ in a humidified incubator containing $5 \% \mathrm{CO}_{2}$ for 2-3 days (permissive condition). To induce differentiation, cells were transferred to RPMI-1640 medium without IFN- $\gamma$ and incubated at $37^{\circ} \mathrm{C}$ in a humidified incubator with $5 \% \mathrm{CO}_{2}$ for 14 days (non-permissive condition).

Construction of recombinant short hairpin (sh)RNA ADAM10 and shCXCL16 interference plasmids. Plasmids were constructed using the lentiviral transferring plasmid, pLKO.1-TRC (pLKO.1; Jiangsu Laisen Biotechnology Co., Ltd.). The sequences of the shADAM10 and shCXCL16 were amplified and inserted into pLKO.1 to generate recombinant pLKO.1-shADAM10 and pLKO.1-shCXCL16 for lentivirus production. The sequences of the forward and reverse primers of the shADAM10 and shCXCL16 are presented in Table I. Using synthetic shADAM10 and shCXCL16 sequences as templates for PCR. PCR amplifications were performed with Phanta Max Super-Fidelity DNA Polymerase (cat. no. DC505; Vazyme Biotech Co., Ltd.), using the following thermocycling conditions: 35 cycles at $95^{\circ} \mathrm{C}$ for $15 \mathrm{sec}, 60^{\circ} \mathrm{C}$ for $30 \mathrm{sec}$ and $72^{\circ} \mathrm{C}$ for $15 \mathrm{sec}$; extension at $72^{\circ} \mathrm{C}$ for $1 \mathrm{~min}$; and maintained at $4^{\circ} \mathrm{C}$. PCR products were verified by $1 \%$ agarose gel electrophoresis and recovered using a purification kit (cat. no. DC301; Vazyme Biotech Co., Ltd.). The restriction enzymes AgeI and EcoRI (New England BioLabs, Inc.) were used to perform a double digest of the plasmid and the PCR gel purification products. The products were separated by $1 \%$ agarose gel electrophoresis, purified and incubated with $\mathrm{T} 4$ ligase overnight at $4^{\circ} \mathrm{C}$. The products were transformed into Escherichia coli, and a single colony was randomly selected. The plasmid was extracted using a plasmid extraction kit (cat. no. DC201; Vazyme Biotech Co., Ltd.). shADAM10 and shCXCL16 recombinant plasmids were verified by a double digest and the products were detected by $1 \%$ agarose gel electrophoresis. The plasmids were subsequently sent to Genscript for sequencing, and the sequencing results were compared with the design sequence.
Production of recombinant lentiviral particles. 293T cells (Conservation Genetics CAS Kunming Cell Bank) were cultured in a $10 \mathrm{~cm}$ cell culture dish at $37^{\circ} \mathrm{C}$ with $5 \% \mathrm{CO}_{2}$ overnight. At $80 \%$ confluence, $293 \mathrm{~T}$ cells were co-transfected with $5.94 \mu \mathrm{g}$ recombinant plasmid $5.94 \mu \mathrm{g}$ pLKO.1-shADAM10, $5.94 \mu \mathrm{g}$ pLKO.1-shCXCL16 or $5.94 \mu \mathrm{g}$ pLKO.1 (empty vector), $4.86 \mu \mathrm{g}$ psPAX2 and $9 \mu \mathrm{g}$ pMD2.G (Jiangsu Laisen Biotechnology Co., Ltd.) using $40 \mu$ l Lipofectamine ${ }^{\circledR} 2000$ (cat. no. 11668019; Thermo Fisher Scientific, Inc.) to produce lentivirus. Similarly, 293T cells were co-transfected with $5.94 \mu \mathrm{g}$ overexpression plasmid pCDH-CMV-ADAM10-Puro (pCDH-ADAM10; Vigene Biosciences), $5.94 \mu \mathrm{g}$ pCDH-CMV-CXCL16-Puro (pCDH-CXCL16; Vigene Biosciences) or $5.94 \mu \mathrm{g} \mathrm{pCDH}$ (empty vector), $4.86 \mu \mathrm{g}$ psPAX2 and $9 \mu \mathrm{g}$ pMD2.G using $40 \mu \mathrm{l}$ Lipofectamine ${ }^{\circledR} 2000$ to produce lentivirus. At $10 \mathrm{~h}$ post-transfection, the cell culture medium was replaced with DMEM medium containing 10\% FBS, $100 \mathrm{U} / \mathrm{ml}$ penicillin and $100 \mu \mathrm{g} / \mathrm{ml}$ streptomycin. At $48 \mathrm{~h}$ post-transfection, the culture medium was collected, filtered through a $0.45-\mu \mathrm{m}$ filter and stored at $-80^{\circ} \mathrm{C}$ until further use.

CXCL16 and ADAM10 overexpression and knockdown in MPC5 cells. CXCL16 and ADAM10 overexpression and knockdown lentiviruses were used to infect MPC5 cells. MPC5 cells $\left(5 \times 10^{5}\right)$ were infected with $200 \mu$ l lentivirus $\left(\sim 2 \times 10^{7}\right.$ viral particles) and $2 \mu \mathrm{l}$ polybrene (Sigma-Aldrich; Merck KGaA). At $48 \mathrm{~h} 37^{\circ} \mathrm{C}$ post-transfection, puromycin $(3 \mu \mathrm{g} / \mathrm{ml})$ was used to screen the cells and surviving cells were used for subsequent experiments.

Reverse transcription-quantitative PCR (RT-qPCR). Total RNA was extracted from cells using TRIzol ${ }^{\circledR}$ reagent (Invitrogen; Thermo Fisher Scientific, Inc.). Total RNA was reverse transcribed into cDNA using a Reverse Transcription system (Vazyme Biotech Co., Ltd.) according to the manufacturer's protocol. The temperature protocol used for reverse transcription was as follows: $50^{\circ} \mathrm{C}$ for $20 \mathrm{~min}$ and $85^{\circ} \mathrm{C}$ for $2 \mathrm{~min}$. Subsequently, qPCR was performed. The sequences of the primers used for qPCR are presented in Table II. The following thermocycling conditions were used for qPCR: $95^{\circ} \mathrm{C}$ for $5 \mathrm{~min}$; 45 cycles of $95^{\circ} \mathrm{C}$ for $10 \mathrm{sec}$ and $60^{\circ} \mathrm{C}$ for $30 \mathrm{sec}$. Relative gene expression was determined by the $2^{-\Delta \Delta \mathrm{Cq}}$ method (18) and normalized to the internal reference gene $\beta$-actin.

Western blotting. Cells were lysed with RIPA lysis buffer (Sangon Biotech Co., Ltd.) supplemented with protease and phosphatase inhibitors. Total protein was quantified using the BCA Protein Assay kit (Beyotime Institute of Biotechnology). Equal quantities of protein $(30 \mu \mathrm{g} / \mathrm{lane})$ were separated via $10 \%$ SDS-PAGE and transferred onto PVDF membranes (EMD Millipore). The membranes were blocked with TBS-T (0.05\% Tween-20) containing 5\% non-fat milk at room temperature for $1 \mathrm{~h}$. Subsequently, the membranes were incubated at $4^{\circ} \mathrm{C}$ overnight with primary antibodies targeted against: $\beta$-actin (1:1,000; cat. no. 3700T; Cell Signaling Technology), CXCL16 (1:1,000; cat. no. 60123-1-Ig; Proteintech), ADAM10 (1:1,000; cat. no. BS60338; Bioworld Technology.) and ACTN4 (1:1,000; cat. no. BS90025; Bioworld Technology). Following primary incubation, the membranes were incubated with horseradish peroxidase-conjugated Affinipure Goat Anti-Rabbit IgG 
Table I. shRNA sequences.

\begin{tabular}{ll} 
shRNA & Sequence $\left(5^{\prime} \rightarrow 3^{\prime}\right)$ \\
\hline shCXCL16-1-F & CCGGGCAGGGTACTTTGGATCACATCTCGAGATGTGATCCAAAGTACCCTGCTTTTTGAATT \\
shCXCL16-1-R & AATTCAAAAAGCAGGGTACTTTGGATCACATCTCGAGATGTGATCCAAAGTACCCTGC \\
shCXCL16-2-F & CCGGGCTGGAAGTTGTTCTTGTGATCTCGAGATCACAAGAACAACTTCCAGCTTTTTGAATT \\
shCXCL16-2-R & AATTCAAAAAGCTGGAAGTTGTTCTTGTGATCTCGAGATCACAAGAACAACTTCCAGC \\
shADAM10-1-F & CCGGGCAGAGAGATACATTAAAGATCTCGAGATCTTTAATGTATCTCTCTGCTTTTTGAATT \\
shADAM10-1-R & AATTCAAAAAGCAGAGAGATACATTAAAGATCTCGAGATCTTTAATGTATCTCTCTGC \\
shADAM10-2-F & CCGGCCAGGAGAGTCTAAGAACTTACTCGAGTAAGTTCTTAGACTCTCCTGGTTTTGAATT \\
shADAM10-2-R & AATTCAAAAACCAGGAGAGTCTAAGAACTTACTCGAGTAAGTTCTTAGACTCTCCTGG
\end{tabular}

sh, short hairpin RNA; CXCL16, CXC motif chemokine ligand 16; ADAM10, ADAM metallopeptidase domain 10; F, forward; R, reverse.

Table II. Primers used for reverse transcription-quantitative PCR.

\begin{tabular}{ll}
\hline Gene & \multicolumn{1}{c}{ Sequence $\left(5^{\prime} \rightarrow 3^{\prime}\right)$} \\
\hline CXCL16- & F: ACCCTTGTCTCTTGCGTTCT \\
Mus-1 & R: GCTTTTGGACTGCAACTGGA \\
CXCL16- & F: ACTCAGCACTCCACTCTTCC \\
Mus-2 & R: ATGGCTGCAGTGAGGAAGAA \\
ADAM10- & F: TTTGTTGAATCTGGCCAGCC \\
Mus-1 & R: TCCCTTCCTTTGCACAGTCA \\
ADAM10- & F: ATTGCTGAGTGGATTGTGGC \\
Mus-2 & R: GATAACTCTCTCGGGGCCTC \\
B-actin-Mus & F: TGGAATCCTGTGGCATCCATGAAAC \\
& R: TAAAACGCAGCTCAGTAACAGTCCG
\end{tabular}

CXCL16, CXC motif chemokine ligand 16; ADAM10, ADAM metallopeptidase domain 10; Mus, mouse; F, forward; R, reverse.

(H+L; 1:5,000; cat. no. SA00001-2; Proteintech) or Affinipure Goat Anti-Mouse IgG (H+L; 1:5,000; cat. no. SA00001-1; Proteintech) secondary antibodies for $1 \mathrm{~h}$ at room temperature. Protein bands were visualized using Western Bright ECL (Thermo Fisher Scientific, Inc.). Protein expression was quantified using Image Lab software (version 4.1; Bio-Rad Laboratories) with $\beta$-actin as the loading control.

Cell migration assay. Cells were treated with oxLDL (50, 80 or $100 \mu \mathrm{g} / \mathrm{ml}$; Beijing Solarbio Science \& Technology Co., Ltd.) for $48 \mathrm{~h}$ at $37^{\circ} \mathrm{C}$. Cell migration was evaluated by performing a Transwell assay (Corning, Inc.). Cells were seeded $\left(5 \times 10^{4}\right.$ cells/well) into the upper chamber with RPMI-1640 without FBS. RPMI-1640 medium containing $10 \%$ FBS was added into the lower chamber. Following incubation at $37^{\circ} \mathrm{C}$ for $12 \mathrm{~h}$, cells on the surface of the Transwell inserts were removed with a cotton swab, and cells on the underside of the insert filter were fixed with $100 \%$ ethyl alcohol and stained with $1 \%$ crystal violet at $24^{\circ} \mathrm{C}$ for $15 \mathrm{~min}$. Stained cells were visualized using a light microscope (magnification, $\mathrm{x} 200$ ).
Oil red $O$ staining. MPC5 cells were treated with oxLDL (50 or $100 \mu \mathrm{g} / \mathrm{ml}$ ) for $48 \mathrm{~h}$ at $37^{\circ} \mathrm{C}$. Subsequently, Oil Red O was dissolved in a small amount of $100 \%$ isopropyl alcohol to a volume of $100 \mathrm{ml}$ and sealed in a brown bottle at $4^{\circ} \mathrm{C}$. Cells were rinsed with PBS, fixed with $10 \%$ neutral formalin for $30 \mathrm{~min}$ at $24^{\circ} \mathrm{C}$, stained with Oil Red $\mathrm{O}$ for $10 \mathrm{~min}$ at $24^{\circ} \mathrm{C}$, rinsed with $60 \%$ isopropyl alcohol and observed under an optical microscope (magnification, $\mathrm{x} 400$ ).

ELISA. The cell culture medium was collected and the levels of released CXCL16 were measured using an ELISA kit (cat. no. AD3057Mo; Andygene) according to the manufacturer's protocol.

Statistical analysis. Data are presented as the mean \pm standard deviation. All experiments were performed in triplicate. Statistical analyses were performed using GraphPad Prism software (version 5.0; GraphPad Software, Inc.). Comparisons among multiple groups were analyzed using one-way ANOVA followed by Tukey's post hoc test. $\mathrm{P}<0.05$ was considered to indicate a statistically significant difference.

\section{Results}

Lipid accumulation is increased in oxLDL-treated cells. MPC5 cells were cultured in RPMI-1640 medium and treated with 50 or $100 \mu \mathrm{g} / \mathrm{ml}$ oxLDL (Beijing Solarbio Science \& Technology Co., Ltd.) for $48 \mathrm{~h}$. Control cells were treated with DMSO. A previous study indicated that $80 \mu \mathrm{g} / \mathrm{ml}$ oxLDL was the most suitable dose (17); therefore, a high dose above the optimal dose $(100 \mu \mathrm{g} / \mathrm{ml})$ and a low dose below the optimal dose $(50 \mu \mathrm{g} / \mathrm{ml})$ were used in the present study. Oil Red O staining was performed to observe intracellular lipid accumulation. The control group displayed a few visible lipid drops (Fig. 1A), whereas a large number of lipid drops were observed in oxLDL-treated cells oxLDL (50 and $100 \mu \mathrm{g} / \mathrm{ml}$; Fig. 1B and C).

oxLDL stimulates podocyte migration. The migratory ability of MPC5 cells was assessed by performing a Transwell assay. The number of migratory cells was significantly increased in a dose-dependent manner in the 50 and $100 \mu \mathrm{g} / \mathrm{ml}$ oxLDL groups compared with the control group (Fig. 2). 
A oxLDL concentration $(0 \mu \mathrm{g} / \mathrm{ml})$

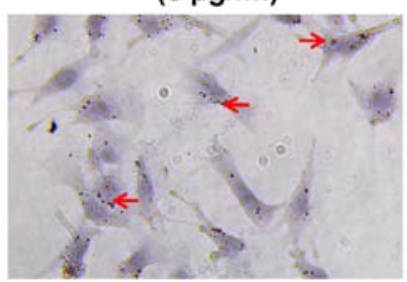

B oxLDL concentration $(50 \mu \mathrm{g} / \mathrm{ml})$

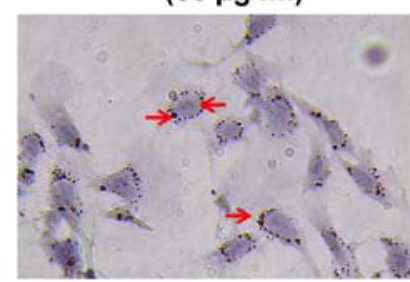

C oxLDL concentration $(100 \mu \mathrm{g} / \mathrm{ml})$

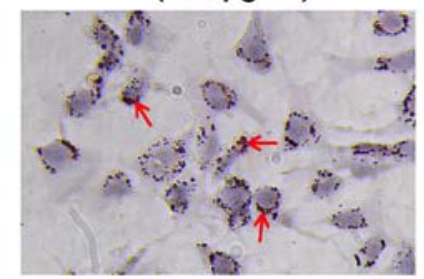

Figure 1. Lipid accumulation in MPC5 cells. (A) Control group. MPC5 cells treated with (B) 50 and (C) $100 \mu \mathrm{g} / \mathrm{ml}$ oxLDL (magnification, x400). The arrows indicate lipid drops. oxLDL, oxidized low-density lipoprotein.
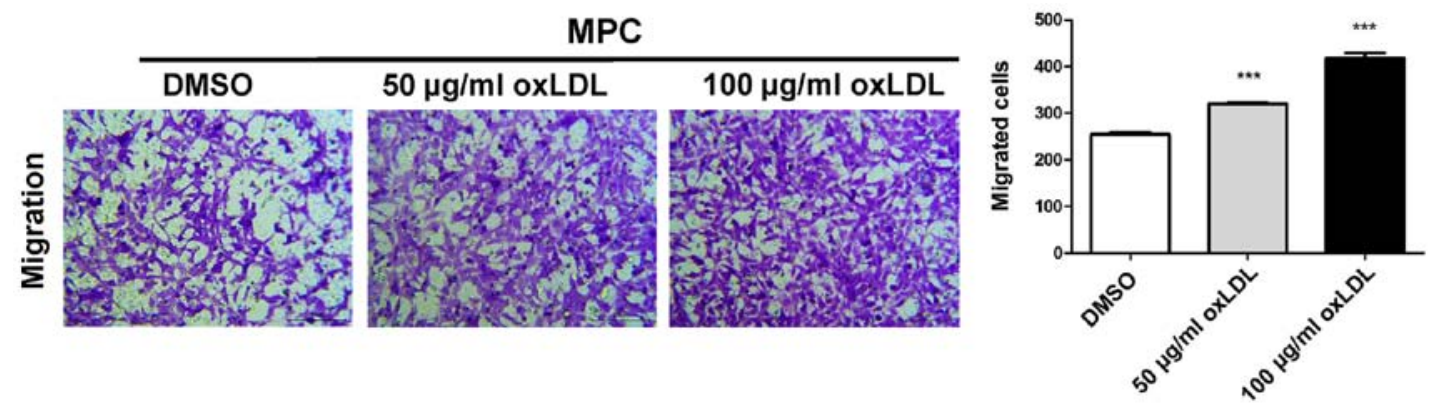

Figure 2. Migratory ability of MPC5 cells treated with oxLDL. The migratory ability of cells was assessed using a Transwell system. oxLDL promoted MPC5 cell migration in a dose-dependent manner. ${ }^{* * *} \mathrm{P}<0.001$ vs. DMSO. oxLDL, oxidized low-density lipoprotein.
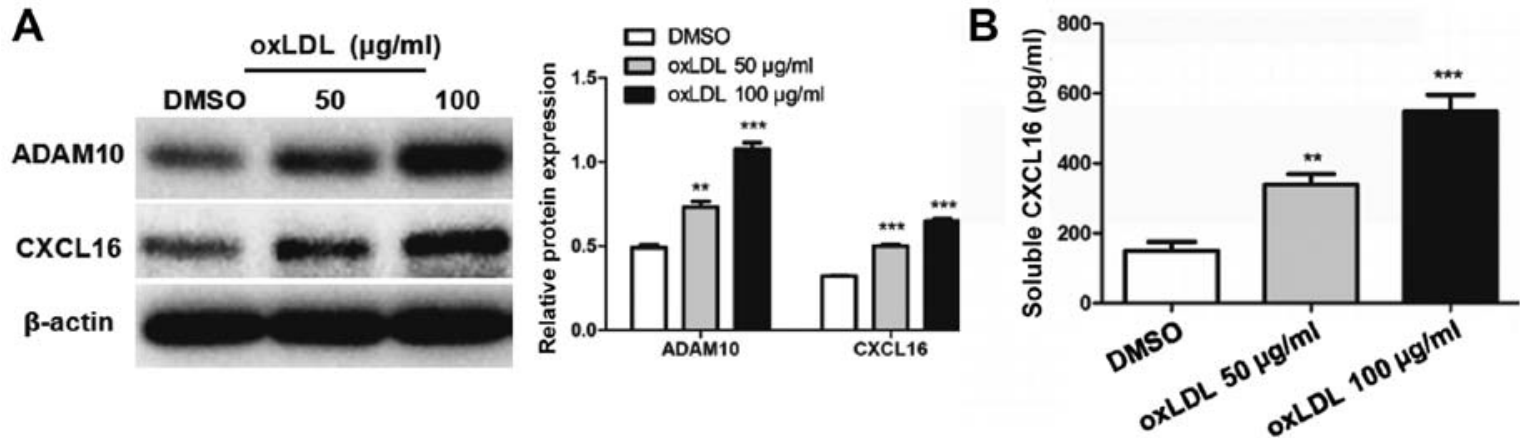

Figure 3. Effect of oxLDL on ADAM10 and CXCL16 expression in MPC5 cells. (A) Protein expression levels of ADAM10 and CXCL16 were detected by western blotting. (B) The level of released CXCL16 in the supernatants was analyzed by performing an ELISA. ${ }^{* *} \mathrm{P}<0.01$ and ${ }^{* * *} \mathrm{P}<0.001 \mathrm{vs}$. DMSO oxLDL, oxidized low-density lipoprotein; ADAM10, ADAM metallopeptidase domain 10; CXCL16, CXC motif chemokine ligand 16.

oxLDL stimulation increases ADAM10 and CXCL16 expression. oxLDL stimulation significantly increased the protein expression levels of ADAM10 and CXCL16 in MPC5 cells compared with DMSO-treated cells (Fig. 3A). Moreover, the level of soluble CXCL16 in the culture medium was significantly increased in the 50 and $100 \mu \mathrm{g} / \mathrm{ml}$ oxLDL groups compared with the control group (Fig. 3B).

Generation of stable CXCL16-and ADAM10-overexpression and knockdown podocyte cell lines. Transfection efficiency was assessed via RT-qPCR. CXCL16 expression was significantly increased by $147.54 \%$ in the overexpression group compared with the pCDH group (Fig. 4A). By contrast, CXCL16 expression was significantly decreased by 79.11 and $80.68 \%$ in the shCXCL16-1 and shCXCL16-2 groups compared with the pLKO.1 group, respectively (Fig. 4B). Similarly, ADAM10 expression was significantly increased by $1,012.07 \%$ in the overexpression group compared with the pCDH group (Fig. 4C), whereas ADAM10 expression was significantly decreased by 68.58 and $66.08 \%$ in the shADAM10-1 and shADAM10-2 groups compared with the pLKO.1 group, respectively (Fig. 4D).

CXCL16 overexpression enhances podocyte migration, whereas CXCL16 knockdown reduces podocyte migration. The migratory ability of CXCL16-overexpression cells was assessed using a Transwell assay. The results indicated that CXCL16 overexpression significantly increased MPC5 cell migration compared with the pCDH group (Fig. 5A). By contrast, CXCL16 knockdown significantly reduced MPC5 cell migration compared with the pLKO-1 group (Fig. 5B).

ADAM10 overexpression enhances podocyte migration, whereas ADAM10 knockdown reduces podocyte migration. 

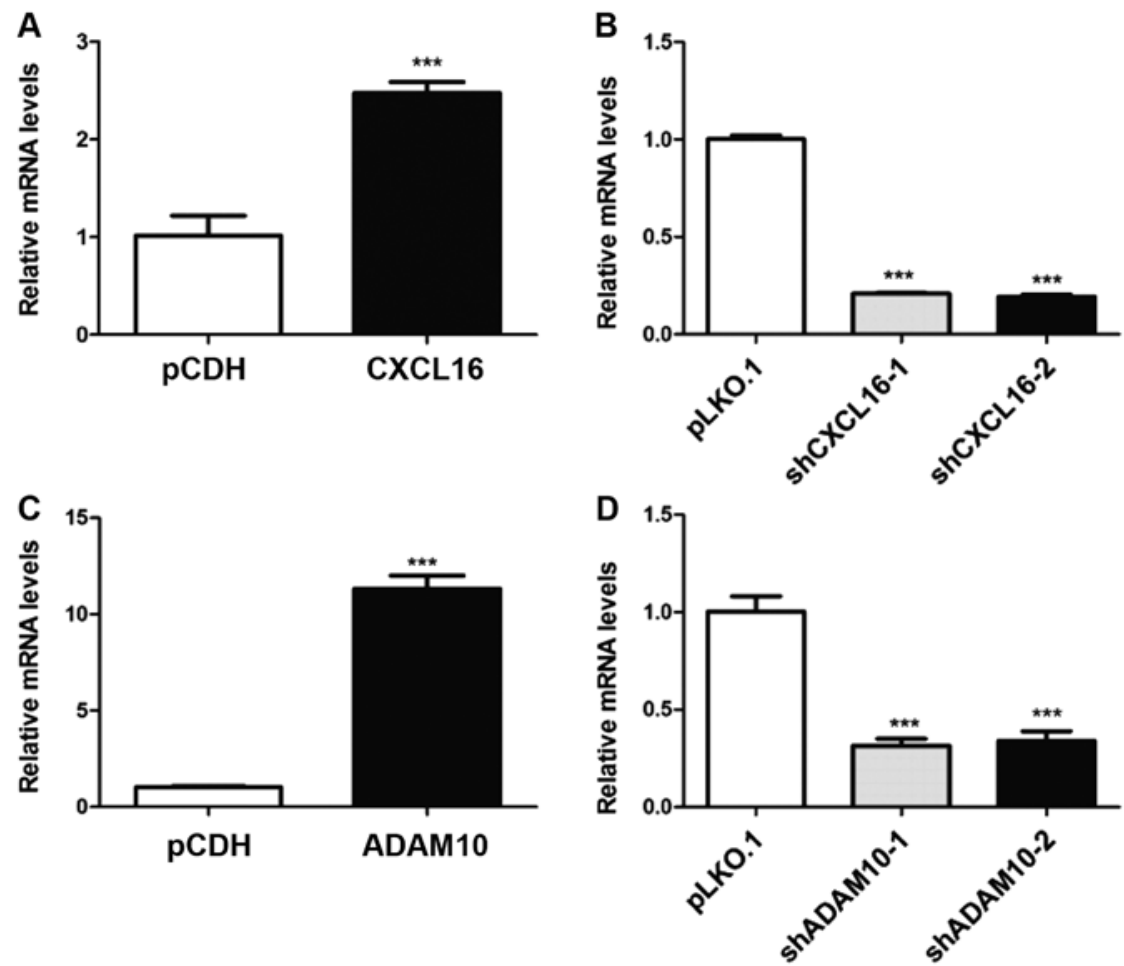

Figure 4. Transfection efficiency of CXCL16 and ADAM10 overexpression and knockdown. CXCL16 overexpression and knockdown successfully (A) increased and (B) decreased the mRNA expression levels of CXCL16, respectively. ADAM10 overexpression and knockdown successfully (C) increased and (D) decreased the mRNA expression levels of ADAM10, respectively. ${ }^{* * *} \mathrm{P}<0.001$ vs. pCDH or pLKO.1. CXCL16, CXC motif chemokine ligand 16; ADAM10, ADAM metallopeptidase domain 10; pCDH, pCDH-EF1-T2A-Puro; pLKO.1, pLKO.1-TRC; sh, short hairpin RNA.

The migratory ability of ADAM10-overexpression cells was assessed using a Transwell assay. The results indicated that ADAM10 overexpression significantly increased MPC5 cell migration compared with the pCDH group (Fig. 6A), whereas ADAM10 knockdown significantly decreased MPC5 cell migration compared with the pLKO.1 group (Fig. 6B).

ADAM10 and CXCL16 protein expression in MPC5 cells. ADAM10-overexpression and -knockdown cells were subjected to western blotting. The expression level of membrane-bound CXCL16wassignificantlydecreasedin ADAM10-overexpression cells compared with pCDH cells (Fig. 7A). Conversely, the expression level of membrane-bound CXCL16 was significantly increased in ADAM10-knockdown cells compared with pLKO.1 cells (Fig. 7C). Soluble CXCL16 release was significantly increased in ADAM10-overexpression cells compared with pCDH cells (Fig. 7B).

CXCL16 and ADAM10 modulate the expression of ACTN4. CXCL16- and ADAM10-overexpression cells displayed significantly increased ACTN4 protein expression levels compared with pCDH cells (Fig. 8A and B). By contrast, CXCL16- and ADAM10-knockdown cells displayed significantly decreased ACTN4 expression levels compared with pLKO.1 cells (Fig. 8C and D).

oxLDL stimulation enhances CXCL16- and ADAM10 knockdown-mediated podocyte migration. Podocyte migration was assessed in CXCL16- and ADAM10-knockdown cells following treatment with $80 \mu \mathrm{g} / \mathrm{ml}$ oxLDL. The control group was treated with DMSO. The results indicated that the number of migratory cells in the oxLDL-treated pLKO.1 group was significantly increased compared with the oxLDL-treated shCXCL16 group. In addition, the number of migratory cells in the DMSO-treated pKLO.1 group was significantly increased compared with the DMSO-treated shCXCL16 group. Moreover, the oxLDL-treated pLKO.1 group displayed significantly increased cell migration compared with the DMSO-treated pLKO.1 group. Similarly, the oxLDL-treated shCXCL16 groupdisplayed significantly increased cell migration compared with the DMSO-treated shCXCL16 group (Fig. 9A). Consistently, the number of migratory cells in the oxLDL-treated pLKO.1 group was significantly increased compared with the oxLDL-treated shADAM10 group. The number of migratory cells in the DMSO-treated pLKO.1 group was significantly increased compared with the DMSO-treated shADAM10 group. Furthermore, the oxLDL-treated pLKO.1 group displayed increased cell migration compared with the DMSO-treated pLKO.1 group and the oxLDL-treated shADAM10 group displayed significantly increased cell migration compared with the DMSO-treated shADAM10 group (Fig. 9B). The results indicated that oxLDL stimulation promoted the migration of CXCL16 and ADAM10-knockdown MPC5 cells.

\section{Discussion}

The present study investigated the effect of oxLDL on podocyte lipid accumulation and migration. The results indicated that oxLDL promoted podocyte lipid accumulation and enhanced MPC5 cell migration in a dose-dependent manner.

NS is a renal disease caused by disruption of the glomerular filtration barrier that results in extensive proteinuria, hypoalbuminemia, hyperlipidemia and edema (1). Based on kidney 

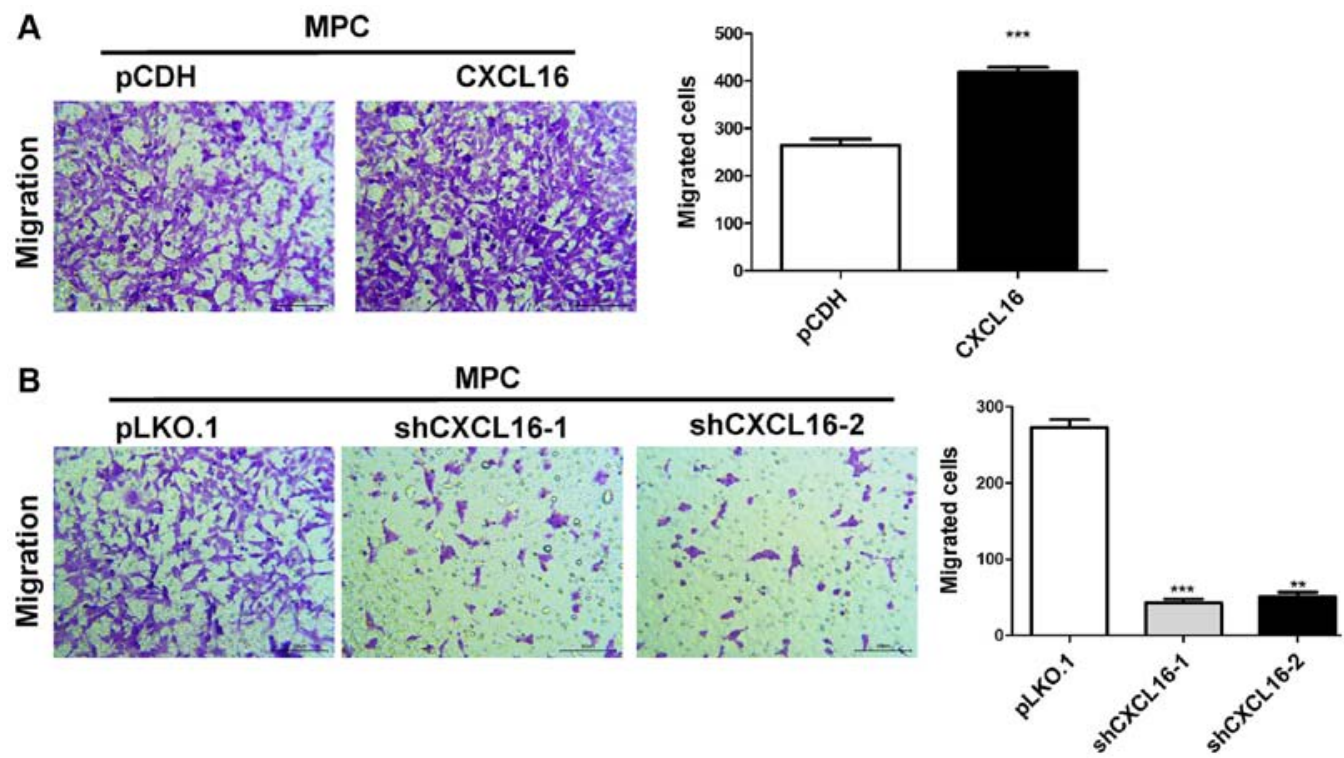

Figure 5. Effect of CXCL16 on podocyte migration. Effect of CXCL16 (A) overexpression and (B) knockdown on podocyte migration. ${ }^{* *} \mathrm{P}<0.01$ and ${ }_{* * * *} \mathrm{P}<0.001$ vs. pCDH or pLKO.1. CXCL16, CXC motif chemokine ligand 16; pCDH, pCDH-EF1-T2A-Puro; pLKO.1, pLKO.1-TRC; sh, short hairpin RNA.

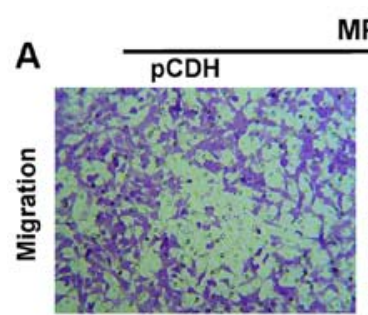

B

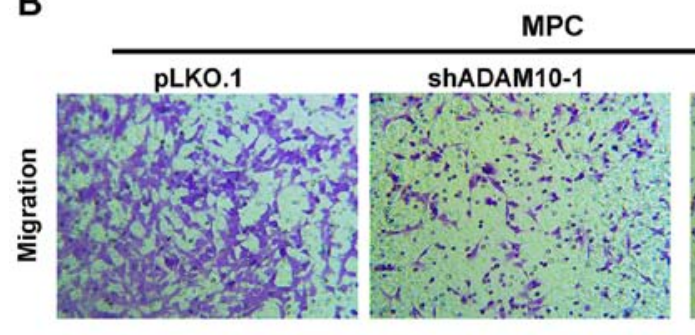

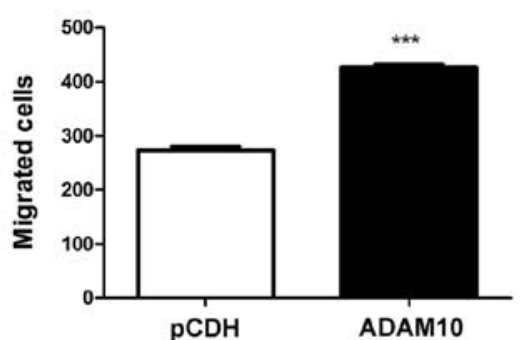

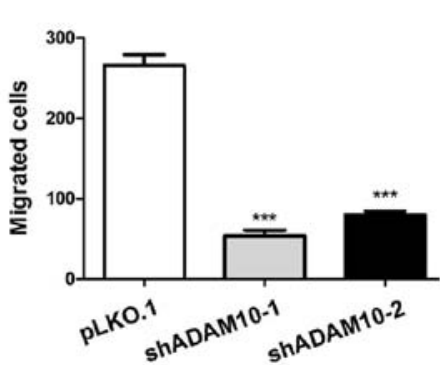

Figure 6. Effect of ADAM10 on podocyte migration. Effect of ADAM10 (A) overexpression and (B) knockdown on podocyte migration. ${ }^{* * *} \mathrm{P}<0.001 \mathrm{vs}$. pCDH or pLKO.1. ADAM10, ADAM metallopeptidase domain 10; pCDH, pCDH-EF1-T2A-Puro; pLKO.1, pLKO.1-TRC; sh, short hairpin RNA.

biopsies, minimal change disease (MCD) is the most common cause of primary NS in children, followed by focal segmental glomerulosclerosis (FSGS) and membranoproliferative glomerulonephritis $(2,3,19)$. Renal biopsies of patients with proteinuria and kidney disease are most often associated with podocyte foot process effacement, which commonly occurs in NS (20).

The podocyte is a highly specialized, terminally differentiated cell that constitutes a crucial component of the glomerular filtration barrier (21-23). Podocyte injury is a hallmark of proteinuria and glomerular diseases $(24,25)$, such as MCD, membranous glomerulopathy, lupus nephritis and diabetic nephropathy (26-28).

Podocytes are a contractile and motile cell type, and their motility must be finely regulated in order to maintain the function of the glomerular filtration barrier (29). Previous studies have suggested that podocytes may transition from a stationary state to a migratory state in response to various stimuli, such as lipopolysaccharide or puromycin aminonucleoside (30-32). Podocyte migration integrates several functions related to adhesion and rearrangement of the actin cytoskeleton (33-35), and increased cell motility could serve as a potential mechanism underlying foot process effacement and proteinuria $(36,37)$. The results of the present study suggested that oxLDL promoted podocyte migration; however, the link between oxLDL-induced migration, and foot process effacement and proteinuria requires further investigation.

The present study also demonstrated that oxLDL stimulation increased the expression of CXCL16 and ADAM10 in podocytes compared with DMSO-treated cells. Furthermore, the levels of both membrane-bound and soluble CXCL16 were increased in oxLDL-stimulated cells compared with control cells. Schramme et al (16) demonstrated that inhibition of ADAM10 and CXCL16 in mesangial cells resulted 

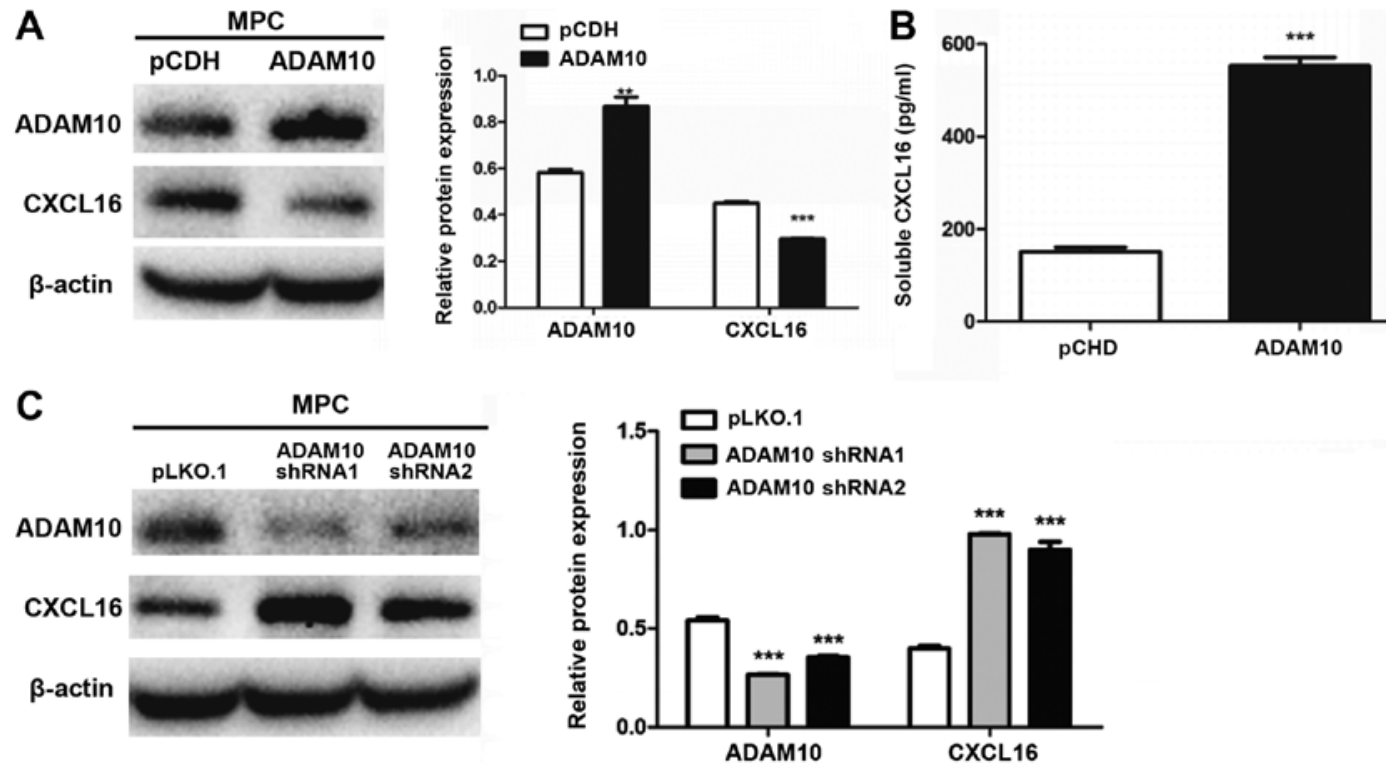

Figure 7. Effect of ADAM10 on CXCL16 expression. Effect of ADAM10 overexpression on the expression levels of (A) membrane-bound and (B) soluble CXCL16. (C) Effect of ADAM10 knockdown on membrane-bound CXCL16 protein expression levels. ${ }^{* *} \mathrm{P}<0.01$ and ${ }^{* * * *} \mathrm{P}<0.001$ vs pCDH or pLKO.1. ADAM10, ADAM metallopeptidase domain 10; CXCL16, CXC motif chemokine ligand 16; pCDH, pCDH-EF1-T2A-Puro; pLKO.1, pLKO.1-TRC; sh, short hairpin RNA.
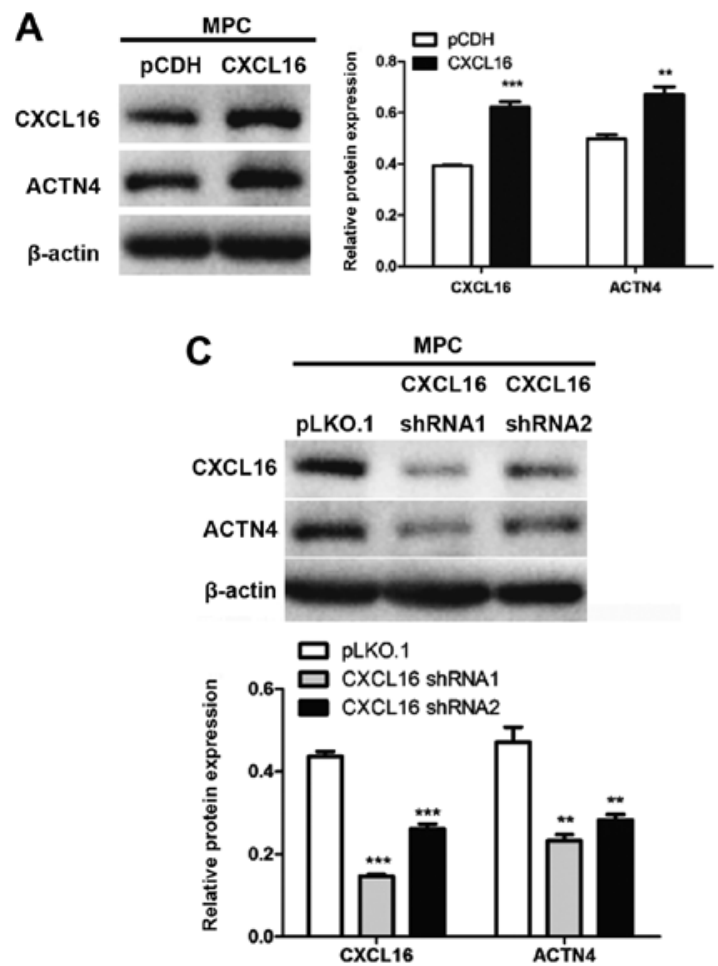
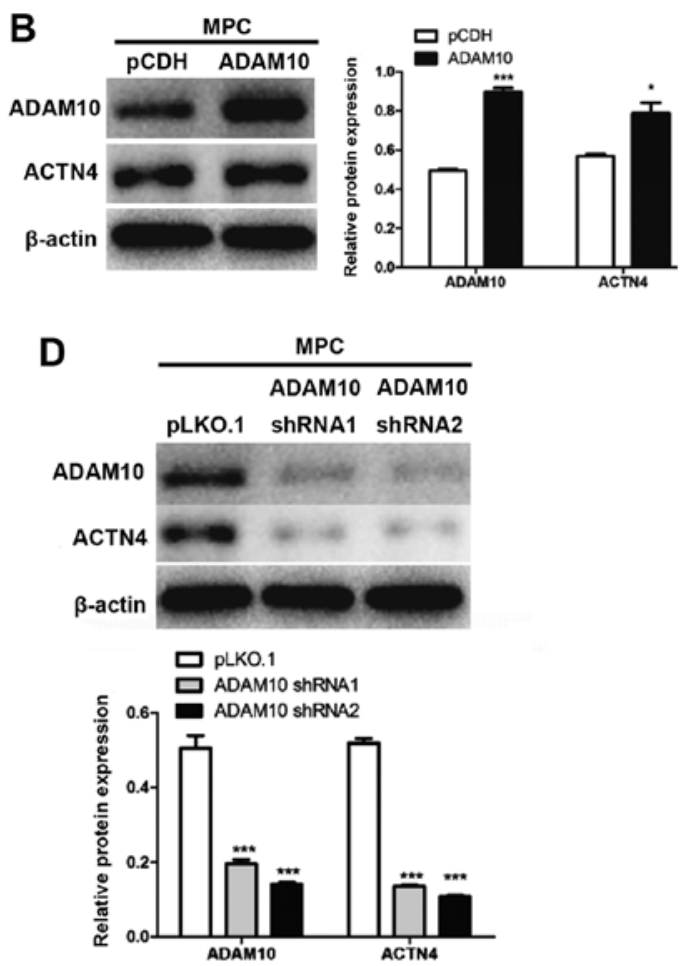

Figure 8. Effect of ADAM10 and CXCL16 on ACTN4 expression. Effect of (A) CXCL16 and (B) ADAM10 overexpression on ACTN4 protein expression levels. Effect of (C) CXCL16 and (D) ADAM10 knockdown on ACTN4 protein expression levels. ${ }^{*} \mathrm{P}<0.05,{ }^{* *} \mathrm{P}<0.01$ and ${ }^{* * * *} \mathrm{P}<0.001$ vs. pCDH or pLKO.1. ADAM10, ADAM metallopeptidase domain 10; CXCL16, CXC motif chemokine ligand 16; ACTN4, actinin- $\alpha 4$; pCDH, pCDH-EF1-T2A-Puro; pLKO.1, pLKO.1-TRC; sh, short hairpin RNA.

in a significant reduction in cell proliferation and migration. Moreover, increasing evidence demonstrates that soluble CXCL16 promotes cancer cell migration in vitro (12).

The present study investigated the effect of CXCL16 and ADAM10 on podocyte migration. CXCL16 and ADAM10 overexpression significantly increased podocyte migration compared with the control groups. Furthermore, podocyte migration was significantly decreased following CXCL16 and ADAM10 knockdown compared with the control groups. CXCL16 exists in two forms in vivo, soluble CXCL16 and membrane-bound CXCL16 (9). ADAM is a $\mathrm{Zn}^{2+}$ dependent transmembrane and metalloproteinase superfamily, known as 'molecular scissors'. ADAM10 is highly expressed in the kidney, where it cleaves the extracellular domain of membrane-bound CXCL16 in a process known as ectodomain shedding, resulting in the release of soluble CXCL16 into 
A

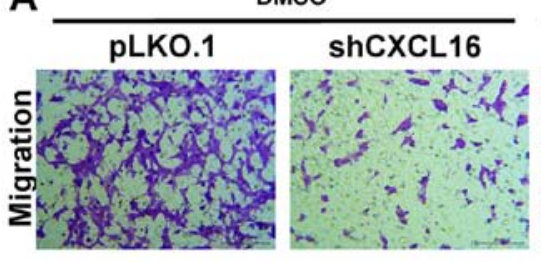

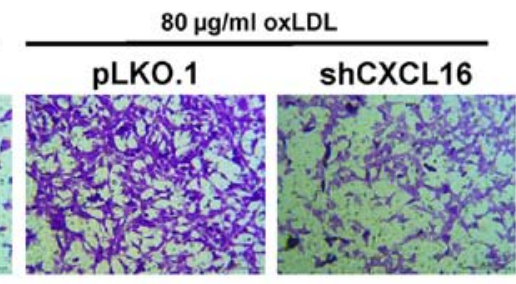

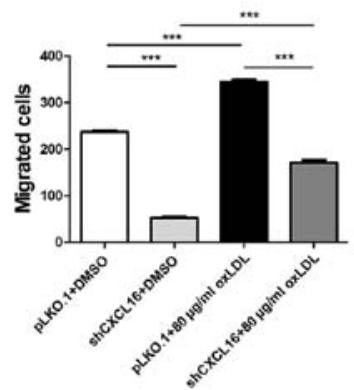

B

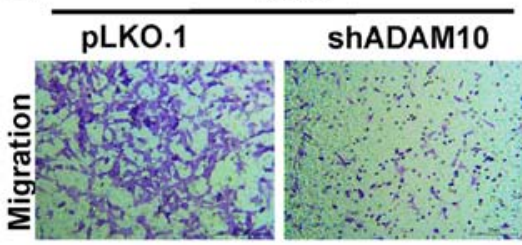

$80 \mu \mathrm{g} / \mathrm{ml}$ oxLDL

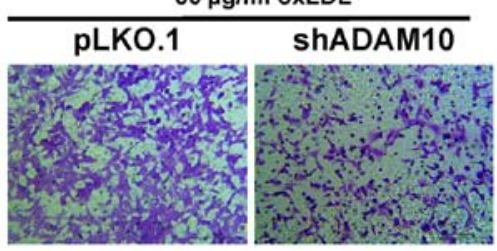

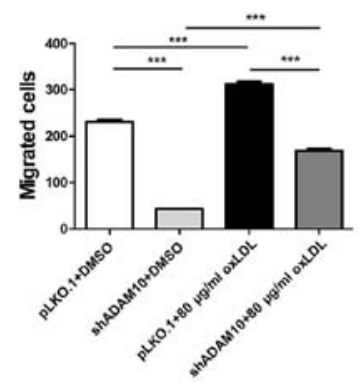

Figure 9. Effect of oxLDL and ADAM10 or CXCL16 knockdown on podocyte migration. Effect of oxLDL on (A) CXCL16 knockdown-mediated podocyte migration and (B) ADAM10 knockdown-mediated podocyte migration. ${ }^{* * *} \mathrm{P}<0.001$. oxLDL, oxidized low-density lipoprotein; ADAM10, ADAM metallopeptidase domain 10; CXCL16, CXC motif chemokine ligand 16; sh, short hairpin RNA; pLKO.1, pLKO.1-TRC.

the circulation (10). ADAM10 overexpression is associated with enhanced release of CXCL16 into culture supernatants and a reduction of membrane-bound protein $(10,15)$. In the present study, membrane-bound CXCL16 expression was decreased in ADAM10-overexpression cells, but was increased in ADAM10-knockdown cells compared with control cells. Moreover, soluble CXCL16 was upregulated in ADAM10-overexpression cells compared with control cells.

In the present study, oxLDL was used to stimulate CXCL16and ADAM10-knockdown podocytes. oxLDL stimulation enhanced CXCL16 and ADAM10 knockdown-mediated cell migration compared with control cells, which suggested that oxLDL promoted podocyte migration by regulating CXCL16 and ADAM10.

ACTN4 is a regulator of the actin cytoskeleton that enables cell migration (38). Several studies have demonstrated that podocyte-specific expression of an FSGS-associated ACTN4 mutant $(\mathrm{K} 256 \mathrm{E})$ results in a reduction in podocyte migration and causes proteinuria in mice $(39,40)$. Wang et al $(41)$ reported that nucleoporin 160 knockdown increased the expression of ACTN4 and enhanced podocyte migration. Therefore, to determine whether CXCL16 and ADAM10 impacted the dynamic actin cytoskeleton rearrangements of podocytes, the present study investigated the expression of ACTN4 in CXCL16- and ADAM10-overexpression and knockdown podocytes. The results revealed that ACTN4 was upregulated in CXCL16- and ADAM10-overexpression cells, but was downregulated in CXCL16- and ADAM10-knockdown cells compared with control cells. Therefore, the regulation of CXCL16 and ADAM10 expression may influence ACTN4 expression, which may result in podocyte migration; however, the link between CXCL16, ADAM10 and ACTN4 requires further investigation.

The present study investigated the effects of oxLDL on podocyte migration. The results revealed that oxLDL enhanced podocyte migration, whereas CXCL16 and ADAM10 mediated the process. More importantly, CXCL16 and ADAM10 overexpression and knockdown significantly increased and decreased podocyte migration compared with control cells, respectively. Furthermore, the results indicated that CXCL16 and ADAM10 may regulate podocyte migration by modulating the actin cytoskeleton. Collectively, the results suggested that oxLDL promoted podocyte migration via regulation of CXCL16 and ADAM10, and modulation of the actin cytoskeleton. Therefore, future studies investigating CXCL16 and ADAM10 are required, as the two proteins may serve as potential therapeutic targets for NS in children.

\section{Acknowledgements}

The authors would like to thank Professor Rong Wang (Shandong Provincial Hospital, Cheeloo College of Medicine, Shandong University) for providing the podocyte cell line.

\section{Funding}

The present study was supported by the Natural Science Foundation of Shandong Province (grant no. ZR2015HM009) and the Shandong Key Research and Development Program (grant no. 2017GSF218005).

\section{Availability of data and materials}

The datasets used and/or analyzed during the current study are available from the corresponding author on reasonable request.

\section{Authors' contributions}

YC and SS designed the present study. YC drafted the manuscript. ZW, QL, LY, YZ and JW evaluated and interpreted the 
data critically. SS revised and approved the final version of the manuscript. All authors read and approved the final manuscript.

\section{Ethics approval and consent to participate}

Not applicable.

\section{Patient consent for publication}

Not applicable.

\section{Competing interests}

The authors declare that they have no competing interests.

\section{References}

1. McCaffrey J, Lennon R and Webb NJ: The non-immunosuppressive management of childhood nephrotic syndrome. Pediatr Nephrol 31: 1383-1402, 2016.

2. Zhao X, Hwang DY and Kao HY: The role of glucocorticoid receptors in podocytes and nephrotic syndrome. Nucl Receptor Res 5: 5, 2018.

3. Ranganathan S: Pathology of podocytopathies causing nephrotic syndrome in children. Front Pediatr 4: 32, 2016.

4. Fornoni A, Merscher S and Kopp JB: Lipid biology of the podocyte - new perspectives offer new opportunities. Nat Rev Nephrol 10: 379-388, 2014.

5. Hu M, Fan M, Zhen J, Lin J, Wang Q, Lv Z and Wang R: FAK contributes to proteinuria in hypercholesterolaemic rats and modulates podocyte $\mathrm{F}$-actin re-organization via activating $\mathrm{p} 38$ in response to ox-LDL. J Cell Mol Med 21: 552-567, 2017.

6. Mertens A and Holvoet P: Oxidized LDL and HDL: Antagonists in atherothrombosis. FASEB J 15: 2073-2084, 2001.

7. Zhang F, Wang C, Wang H, Lu M, Li Y, Feng H, Lin J, Yuan Z and Wang $\mathrm{X}$ : Ox-LDL promotes migration and adhesion of bone marrow-derived mesenchymal stem cells via regulation of MCP-1 expression. Mediators Inflamm 2013: 691023, 2013.

8. Rubina K, Talovskaya E, Cherenkov V, Ivanov D, Stambolsky D, Storozhevykh T, Pinelis V, Shevelev A, Parfyonova Y Resink T, et al: LDL induces intracellular signalling and cell migration via atypical LDL-binding protein T-cadherin. Mol Cell Biochem 273: 33-41, 2005.

9. Liang H, Liao M, Zhao W, Zheng X, Xu F, Wang $\mathrm{H}$ and Huang J: CXCL16/ROCK1 signaling pathway exacerbates acute kidney injury induced by ischemia-reperfusion. Biomed Pharmacother 98: 347-356, 2018.

10. Kato T, Hagiyama M and Ito A: Renal ADAM10 and 17: Their physiological and medical meanings. Front Cell Dev Biol 6: 153, 2018.

11. Hu ZB, Chen Y, Gong YX, Gao M, Zhang Y, Wang GH, Tang RN, Liu H, Liu BC and Ma KL: Activation of the CXCL16/CXCR6 pathway by inflammation contributes to atherosclerosis in patients with end-stage renal disease. Int J Med Sci 13: 858-867, 2016.

12. Fang Y, Henderson FC Jr, Yi Q, Lei Q, Li Y and Chen N: Chemokine CXCL16 expression suppresses migration and invasiveness and induces apoptosis in breast cancer cells. Mediators Inflamm 2014: 478641, 2014

13. Garcia GE, Truong LD, Li P, Zhang P, Johnson RJ, Wilson CB and Feng L: Inhibition of CXCL16 attenuates inflammatory and progressive phases of anti-glomerular basement membrane antibody-associated glomerulonephritis. Am J Pathol 170: 1485-1496, 2007.

14. Schramme A,Abdel-Bakky MS, Gutwein P, Obermüller N,Baer PC, Hauser IA, Ludwig A, Gauer S, Schäfer L, Sobkowiak E, et al: Characterization of CXCL16 and ADAM10 in the normal and transplanted kidney. Kidney Int 74: 328-338, 2008.

15. Gutwein P, Abdel-Bakky MS, Schramme A, Doberstein K, Kämpfer-Kolb N, Amann K, Hauser IA, Obermüller N, Bartel C, Abdel-Aziz AA, et al: CXCL16 is expressed in podocytes and acts as a scavenger receptor for oxidized low-density lipoprotein. Am J Pathol 174: 2061-2072, 2009.
16. Schramme A, Abdel-Bakky MS, Kämpfer-Kolb N, Pfeilschifter J and Gutwein P: The role of CXCL16 and its processing metalloproteinases ADAM10 and ADAM17 in the proliferation and migration of human mesangial cells. Biochem Biophys Res Commun 370: 311-316, 2008.

17. Wang L, Sun S, Zhou A, Yao X and Wang Y: oxLDL-induced lipid accumulation in glomerular podocytes: Role of IFN- $\gamma$, CXCL16, and ADAM10. Cell Biochem Biophys 70: 529-538, 2014.

18. Livak KJ and Schmittgen TD: Analysis of relative gene expression data using real-time quantitative PCR and the 2(-Delta Delta C(T)) method. Methods 25: 402-408, 2001.

19. Davin JC: The glomerular permeability factors in idiopathic nephrotic syndrome. Pediatr Nephrol 31: 207-215, 2016.

20. Kalluri R: Proteinuria with and without renal glomerular podocyte effacement. J Am Soc Nephrol 17: 2383-2389, 2006.

21. Assady S, Wanner N, Skorecki KL and Huber TB: New insights into podocyte biology in glomerular health and disease. J Am Soc Nephrol 28: 1707-1715, 2017.

22. Reiser J and Altintas M: Podocytes. F1000Res 5: 114, 2016.

23. Tan X, Chen Y, Liang X, Yu C, Lai Y, Zhang L, Zhao X, Zhang H, Lin T, Li R, et al: Lipopolysaccharide-induced podocyte injury is mediated by suppression of autophagy. Mol Med Rep 14: 811-818, 2016

24. Greka A and Mundel P: Cell biology and pathology of podocytes. Annu Rev Physiol 74: 299-323, 2012.

25. Lin T, Zhang L, Liu S, Chen Y, Zhang H, Zhao X, Li R, Zhang Q, Liao R, Huang Z, et al: WWC1 promotes podocyte survival via stabilizing slit diaphragm protein dendrin. Mol Med Rep 16: 8685-8690, 2017.

26. Akchurin O and Reidy KJ: Genetic causes of proteinuria and nephrotic syndrome: Impact on podocyte pathobiology. Pediatr Nephrol 30: 221-233, 2015.

27. Yu SM, Nissaisorakarn P, Husain I and Jim B: Proteinuric kidney diseases: A Podocyte's slit diaphragm and cytoskeleton approach. Front Med (Lausanne) 5: 221, 2018.

28. Vallon V and Komers R: Pathophysiology of the diabetic kidney. Compr Physiol 1: 1175-1232, 2011.

29. Noris M and Remuzzi G: Non-muscle myosins and the podocyte. Clin Kidney J 5: 94-101, 2012.

30. Jin X, Wang W, Mao J, Shen H, Fu H, Wang X, Gu W, Liu A, Yu H, Shu Q, et al: Overexpression of Myole in mouse podocytes enhances cellular endocytosis, migration, and adhesion. J Cell Biochem 115: 410-419, 2014.

31. Kistler AD, Altintas MM and Reiser J: Podocyte GTPases regulate kidney filter dynamics. Kidney Int 81: 1053-1055, 2012.

32. Cechova S, Dong F, Chan F, Kelley MJ, Ruiz P and Le TH: MYH9 E1841K mutation augments proteinuria and podocyte injury and migration. J Am Soc Nephrol 29: 155-167, 2018.

33. Shao H, Wang JH, Pollak MR and Wells A: $\alpha$-actinin-4 is essential for maintaining the spreading, motility and contractility of fibroblasts. PLoS One 5: e13921, 2010.

34. Duning K, Schurek EM, Schlüter M, Bayer M, Reinhardt HC, Schwab A, Schaefer L, Benzing T, Schermer B, Saleem MA, et al: KIBRA modulates directional migration of podocytes. J Am Soc Nephrol 19: 1891-1903, 2008.

35. Fernández D, Horrillo A, Alquezar C, González-Manchón C, Parrilla R and Ayuso MS: Control of cell adhesion and migration by podocalyxin. Implication of Rac1 and $\mathrm{Cdc} 42$. Biochem Biophys Res Commun 432: 302-307, 2013.

36. Mundel P and Reiser J: Proteinuria: An enzymatic disease of the podocyte? Kidney Int 77: 571-580, 2010.

37. Li Z, Zhang L, Shi W, Chen Y, Zhang H, Liu S, Liang X, Ling T, Yu C, Huang Z, et al: Spironolactone inhibits podocyte motility via decreasing integrin $\beta 1$ and increasing integrin $\beta 3$ in podocytes under high-glucose conditions. Mol Med Rep 12: 6849-6854, 2015.

38. Shao H, Travers T, Camacho CJ and Wells A: The carboxyl tail of alpha-actinin-4 regulates its susceptibility to m-calpain and thus functions in cell migration and spreading. Int $\mathrm{J}$ Biochem Cell Biol 45: 1051-1063, 2013.

39. Michaud JL, Chaisson KM, Parks RJ and Kennedy CR: FSGS-associated alpha-actinin-4 (K256E) impairs cytoskeletal dynamics in podocytes. Kidney Int 70: 1054-1061, 2006.

40. Feng D, DuMontier C and Pollak MR: The role of alpha-actinin-4 in human kidney disease. Cell Biosci 5: 44, 2015.

41. Wang P, Zhao F, Nie X, Liu J and Yu Z: Knockdown of NUP160 inhibits cell proliferation, induces apoptosis, autophagy and cell migration, and alters the expression and localization of podocyte associated molecules in mouse podocytes. Gene 664: 12-21, 2018.

This work is licensed under a Creative Commons Attribution-NonCommercial-NoDerivatives 4.0 International (CC BY-NC-ND 4.0) License. 